\title{
Predição de modelos sobre a cinética de secagem de folhas de Ziziphus joazeiro Mart
}

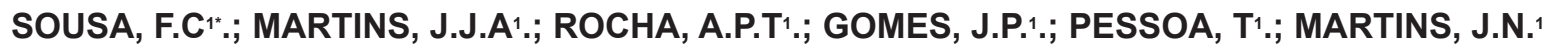

Departamento de Engenharia Agrícola, Universidade Federal de Campina Grande, Caixa Postal 10.078, CEP: 58429-140, Campina Grande-Brasil; francis_nalva@yahoo.com.br

\begin{abstract}
RESUMO: Esta pesquisa objetivou estudar, experimentalmente, a cinética de secagem de folhas de juazeiro (Ziziphus joazeiro Mart.) sob camada delgada em estufa com circulação forçada de ar utilizando temperaturas de 40,50 e $60^{\circ} \mathrm{C}$ e velocidade do ar de $0,5,1,0$ e 1,5 m.s $\mathrm{s}^{-1}$ conforme planejamento fatorial $\left(2^{2}+3\right)$ e posterior ajuste das equações matemáticas aos dados experimentais, verificando assim aquele que melhor representa o fenômeno de secagem. Foi determinado o teor de água inicial das folhas utilizando o método padrão da estufa, em triplicatas. Para cada tratamento de secagem foram utilizados em torno de $150 \mathrm{~g}$ de folhas. Para o ajuste utilizou-se análise de regressão não linear, pelo método Quasi-Newton, por meio do programa computacional Statistica $5.0^{\circledR}$, em que os valores dos parâmetros das equações foram estimados em função da temperatura e da velocidade do ar de secagem. A equação que melhor representou o processo de secagem do juazeiro para a faixa de temperatura de 40 a $60{ }^{\circ} \mathrm{C}$ foi a de Midilli. Os resultados mostraram que com o acréscimo da temperatura ocorre maior redução no tempo de secagem.
\end{abstract}

Palavras-chave: Secagem, Ziziphus joazeiro Mart, modelagem.

\begin{abstract}
Prediction models in the kinetics of drying leaves of Ziziphus joazeiro Mart. This research aimed to study experimentally the kinetics of drying leaves of Ziziphus joazeiro Mart. in thin layer in an oven with forced air using the temperatures of 40,50 and $60^{\circ} \mathrm{C}$ and the air velocity of $0.5,1.0$ and $1.5 \mathrm{~m} \mathrm{~s}^{-1}$ according to the factorial design $\left(2^{2}+3\right)$ and subsequent adjustment of the experimental data in different models, and we select the one that best represents the phenomenon of drying. We determined the initial water content of the leaves by the standard oven method, in triplicates. For each drying treatment, we used approximately $150 \mathrm{~g}$ of leaves. For adjustment, we used the non-linear regression analysis, through the QuasiNewton method, using the software Statistica $5.0 \AA$, in which the parameter values of the equations were determined for the temperature and velocity of the drying air. The equation that best represented the drying process of the Ziziphus joazeiro Mart. for the temperature range of $40-60^{\circ} \mathrm{C}$ was the Midilli equation. The results showed that there is a greater reduction in drying time with increasing temperature.
\end{abstract}

Key words: drying, Ziziphus joazeiro Mart., modeling

\section{INTRODUÇÃO}

O Juazeiro (Ziziphus joazeiro Mart), pertencente à família Rhamanacea, conhecido popularmente como juá, é uma árvore típica do Nordeste brasileiro, caracteriza-se por ser uma espécie endêmica da caatinga, de médio porte, podendo resistir as grandes secas, permanecendo sempre verde. Apesar de ser uma espécie bastante utilizada pela população do semiárido nordestino, a exploração do juazeiro limita-se ao extrativismo predatório (LOPPES, 2008). A secagem é o processo comercial bastante utilizado para a preservação da qualidade dos produtos agrícolas. Consiste na remoção de grande parte de água inicialmente contida no produto logo após a maturidade fisiológica, para que promova longos períodos de armazenamento, sem que ocorram perdas significativas durante o processo. Na secagem de plantas, conforme a espécie, as partes frescas colhidas devem ser preparadas para o processo, visando aumentar a eficiência (Martinazzo, 2010).

De acordo com Martinazzo (2010) a secagem com ar aquecido e com uma baixa temperatura é a técnica mais utilizada em regiões de clima úmido e chuvoso. O aquecimento pode ser realizado em secadores ou estufas, no qual o tempo de secagem é bastante reduzido usando 
estes equipamentos. Os limites de temperaturas do ar de secagem são determinados em função da sensibilidade dos compostos químicos presentes nas plantas e das estruturas armazenadoras.

$O$ estudo e a modelagem matemática tem despertado o interesse de vários pesquisadores para os mais diversos produtos (Faria, 2012). Neste sentido Randuz et al. (2011) retratam a importância do ajuste de diferentes modelos matemáticos aos dados experimentais de secagem, para as mais diversas espécies de plantas medicinais, aromáticas e condimentares, a fim se obter o modelo mais adequado para cada espécie vegetal.

As curvas de secagem em camada delgada variam com a espécie, variedade, condições ambientais e métodos de preparo pós-colheita, entre outros fatores. Esses modelos, geralmente, baseiam-se em variáveis externas ao produto, como a temperatura e a umidade relativa do ar de secagem (Resende et al., 2008).

Objetivou-se, no presente trabalho, ajustar modelos matemáticos aos dados experimentais de secagem de folhas de juazeiro (Zizhipus joazeiro Mart) em diferentes temperaturas e velocidade do ar de secagem.

\section{MATERIAL E MÉTODOS}

Foram utilizadas para a realização da cinética de secagem folhas de juazeiro (Ziziphus joazeiro Mart.) provenientes da Universidade Federal de Campina Grande (UFCG). As folhas foram coletadas manualmente pela manhã no horário das 7:00 as 8:00 h, apresentando teor de água em torno de $58 \%$ (base úmida). O material foi encaminhado ao Laboratório de Química e Biomassa (LBQ) da Universidade Federal de Campina Grande (UFCG) para seleção, determinação do teor de água e secagem em estufa com circulação forçada de ar.

Após a colheita, antes do processo de secagem, o material foi transportado ao laboratório acondicionado em sacos de polietileno, onde foram selecionadas para a retirada de partes danificadas. Depois do processo de seleção as mesmas tiveram suas extremidades inferiores e superiores cortadas manualmente, havendo o cuidado de trabalhar somente com a parte central. As plantas foram cortadas de maneira transversal em pedaços de no máximo $2 \mathrm{~cm}$ de espessura. Após esta etapa as plantas foram homogeneizadas e colocadas em uma cesta de material metálico, em tela de arame de malha fina.

O teor de água inicial do produto foi determinado pelo método gravimétrico, utilizandose estufa a $105 \pm 3{ }^{\circ} \mathrm{C}$, durante $24 \mathrm{~h}$, até peso constante, em três repetições de acordo com Brasil
(2005).

Após a homogeneização do material, o conjunto (cesta + amostra) foi pesado em balança semi-analítica com precisão de 0,01g e colocada na estufa (câmara de secagem), procedendo ao inicio da operação. Após o equipamento ter sido ligado, foi determinada a velocidade do ar, através de um anemômetro digital de pás rotativas modelo Thal-300 da Instrutherm, com precisão de $\pm 3 \%$, colocado na lateral da estufa, bem como o ajuste das temperaturas a serem trabalhadas.

A secagem foi realizada em dias alternados em camada delgada em estufa com circulação de ar com temperatura e velocidade do ar controlada (40, 50 e $60^{\circ} \mathrm{C}$ e $0,5,1,0$ e $1,5 \mathrm{~m} \mathrm{~s}^{-1}$ ) e a umidade relativa do ar monitorada através de um termo-higrômetro digital (modelo TH-439 da Equitherm). O teor de água perdido durante o processo de secagem foi obtido por pesagens descontínuas das amostras e o esquema das pesagens seguiu intervalos de $5,10,20,30$ e $60 \mathrm{~min}$, até o final do processo. As pesagens foram realizadas até que as variações de massa fossem insignificantes. A secagem foi concluída quando não havia variação do peso na ordem de 0,01 g entre três pesagens sucessivas.

Para análise de regressão não linear dos dados experimentais, utilizou-se um planejamento fatorial $2^{2}$ com três repetições no ponto central (nível 0) empregando-se o programa computacional Statistica, versão 5.0, conforme Tabela 3.1. Os dados relativos ao planejamento encontram-se na Tabela 1. As variáveis estudadas foram a temperatura e a velocidade do ar de secagem. Os experimentos foram realizados em forma aleatória, visando evitar erros estatísticos que comprometa a qualidades dos resultados obtidos.

TABELA 1. Matriz experimental do planejamento fatorial

\begin{tabular}{ccc}
\hline Ensaios & $\begin{array}{c}\text { Velocidade do ar } \\
\left(\mathbf{m ~ s}^{-1}\right)\end{array}$ & $\begin{array}{c}\text { Temperatura } \\
\left({ }^{\circ} \mathbf{C}\right)\end{array}$ \\
\hline $\mathbf{1}$ & $0,5(-)$ & $40(-)$ \\
$\mathbf{2}$ & $0,5(-)$ & $60(-)$ \\
$\mathbf{3}$ & $1,5(+)$ & $40(+)$ \\
$\mathbf{4}$ & $1,5(+)$ & $60(+)$ \\
$\mathbf{5}$ & $1,0(0)$ & $50(0)$ \\
$\mathbf{6}$ & $1,0(0)$ & $50(0)$ \\
$\mathbf{7}$ & $1,0(0)$ & $50(0)$ \\
\hline
\end{tabular}

Para determinação da razão de teor de água das folhas de juazeiro para as diferentes condições de secagem foi utilizada a seguinte equação:

$$
\mathrm{RX}=\frac{\mathrm{X}_{\mathrm{bs}}-\mathrm{X}_{\mathrm{e}}}{\mathrm{X}_{\mathrm{bs} \text { inicial }}-\mathrm{X}_{\mathrm{e}}}
$$


Onde:

$\mathrm{RX}$ - razão de umidade, (adimensional)

$\mathrm{Xe}$ - teor de água de equilíbrio (b.s.)

Xbs - teor de água (b.s.)

Os valores de RX observados para cada temperatura do ar de secagem foram analisados por dez diferentes equações empíricas e semiempíricas e regressão não linear, conforme a Tabela 2.

Para o ajuste dos modelos matemáticos aos dados experimentais, realizou-se análise de regressão não linear, pelo método Quase-Newton, empregando-se o programa computacional Statistica 5.0®. Os critérios usados para determinação do melhor ajuste dos modelos aos dados experimentais foi o coeficiente de determinação $\left(R^{2}\right)$ e o desvio quadrático médio (DQM), calculado pela Equação 12.

$\mathrm{DQM}=\sqrt{\frac{\sum\left(\mathrm{RX}_{\exp }-\mathrm{RX}_{\mathrm{pre}}\right)^{2}}{\mathrm{~N}}}$

em que:

DQM - desvio quadrático médio;

$\mathrm{RX}_{\text {pre }}$ - razão de umidade predito;

$\mathrm{RX}_{\text {exp }}$ - razão de umidade experimental;

$\mathrm{N}$ - número de dados experimentais.

\section{RESULTADOS E DISCUSSÃO}

$\mathrm{Na}$ Figura 1 encontram-se as curvas de secagem das folhas de juazeiro, obtida sob diferentes condições de temperatura (40,50 e 60 $\left.{ }^{\circ} \mathrm{C}\right)$ e velocidade do ar de secagem $(0,5,1,0$ e 1,5 $\mathrm{m} \mathrm{s}^{-1}$ ), conforme a matriz experimental apresentada na Tabela 1.

Observou-se na Figura 1 que, quanto mais elevada à temperatura, mais rapidamente se processou a secagem. Quanto maior for a temperatura do ar, maior é a taxa de secagem. No inicio da secagem observa-se que a taxa de secagem é constante e que a partir de aproximadamente 250 minutos esta taxa começou a decrescer. Os tempos médios necessários para completar o processo de secagem foram de 945, 675 e 555 respectivamente, cujos resultados estão semelhantes com os estudos realizados por Randuz et al. (2010) e Martinazzo et al. (2007) quando pesquisaram sobre a cinética de secagem de folhas de sálvia (Salvia officinalis $L$ ) e folhas de capim-limão (Cymbopogon citratus).

Quanto à velocidade do ar de secagem, os resultados deixaram claro que esta teve uma pequena influência sobre a taxa de secagem das folhas de juazeiro, quando se trabalhou com a velocidade de $1,5 \mathrm{~m} \mathrm{~s}^{-1}$, contribuindo para que 0 processo de secagem ocorresse de forma mais rápida. Para as curvas referentes ao ponto central $\left(50^{\circ} \mathrm{C}\right)$ da matriz experimental, observou-se a boa reprodutibilidade dos dados. As curvas de $50^{\circ} \mathrm{C}$ e $1 \mathrm{~m} \mathrm{~s}^{-1}$ apresentam tendência semelhante, não havendo superposição das mesmas devido ao fato de que as amostras foram secas em dias alternados e que variáveis como umidade relativa do ambiente e teor de água inicial das mesmas são incontroláveis. Resultados semelhantes foram observados por Dias et al. (2011) que estudando a secagem em camada delgada e a extração de taninos totais de hortelã (Mentha $x$ vilosa Hudson) observaram o mesmo comportamento para as curvas referentes ao ponto central.

Na Tabela 3 está apresentado o resumo do ajuste dos dez modelos analisados por meio de regressão não linear aos dados experimentais da secagem de folhas de juazeiro em camada delgada, considerando as diferentes temperaturas e velocidade do ar de secagem.

TABELA 2. Modelos matemáticos de regressão não linear, avaliados para predizer o fenômeno de secagem de folhas de juazeiro.

\begin{tabular}{lllc}
\hline Designação do modelo & Equação & Referência & Designação \\
\hline Aprox. por difusão & $\mathrm{RU}=\mathrm{a} \cdot \exp (-\mathrm{k} \cdot \mathrm{t})+(1-\mathrm{a}) \cdot \exp (-\mathrm{k} \cdot \mathrm{b} \cdot \mathrm{t})$ & Corrêa et al, (2010) & $(2)$ \\
Cavalcanti Mata & $\mathrm{RU}=\mathrm{a} 1 \cdot \exp ((\mathrm{a} 2 \cdot(\mathrm{t} \mathrm{a}))+\mathrm{a} 4 \cdot \exp (\mathrm{a} 2 \cdot(\mathrm{ta}))+\mathrm{a} 6$ & Cavalcanti Mata $(2006)$ & $(3)$ \\
Dois termos & $\mathrm{RU}=\mathrm{a} \cdot \exp (-\mathrm{k} \cdot \mathrm{t})+\mathrm{b} \cdot \exp (-\mathrm{k} \cdot \mathrm{t})$ & Jittanit $(2011)$ & $(4)$ \\
Exponencial de dois termos & $\mathrm{RU}=\mathrm{a} \cdot \exp (-\mathrm{k} \cdot \mathrm{t})+(1-\mathrm{a}) \exp (-\mathrm{k} \cdot \mathrm{a} \cdot \mathrm{t})$ & $($ SHARAF-ELDEE et al., 1980) & $(5)$ \\
Henderson \& Pabis Modificado & $\mathrm{RU}=\mathrm{a} \cdot \exp \left(-\mathrm{k}_{0} \cdot \mathrm{t}\right)+\exp \left(-\mathrm{k}_{1} \cdot \mathrm{t}\right)$ & Henderson \& Pabis $(1961)$ & $(6)$ \\
Logarítmico & $\mathrm{RU}=\mathrm{a} \cdot \exp (-\mathrm{k} \cdot \mathrm{t})+\mathrm{c}$ & (YAGCIOGLU et al., 1999) & $(7)$ \\
Midilli et al. & $\mathrm{RU}=\mathrm{a} \cdot \exp (-\mathrm{k} \cdot \mathrm{t} \mathrm{n})+\mathrm{b} \cdot \mathrm{t}$ & Midilli et al. $(2002)$ & $(8)$ \\
Page & $\mathrm{RU}=\exp (-\mathrm{k} \cdot \mathrm{t})$ & (PAGE, 1949) & $(9)$ \\
Verma & $\mathrm{RU}=\mathrm{a} \cdot \exp (-\mathrm{k} \cdot \mathrm{t})+(1-\mathrm{a}) \exp \left(-\mathrm{k}_{1} \cdot \mathrm{t}\right)$ & (VERMA et al., 1985) & $(10)$ \\
Wang e Sing & $\mathrm{RU}=1+\mathrm{a} \cdot \mathrm{t}+\mathrm{b} \cdot \mathrm{t} 2$ & & $(11)$ \\
\hline
\end{tabular}

Rev. Bras. PI. Med., Campinas, v.17, n.2, p.195-200, 2015. 


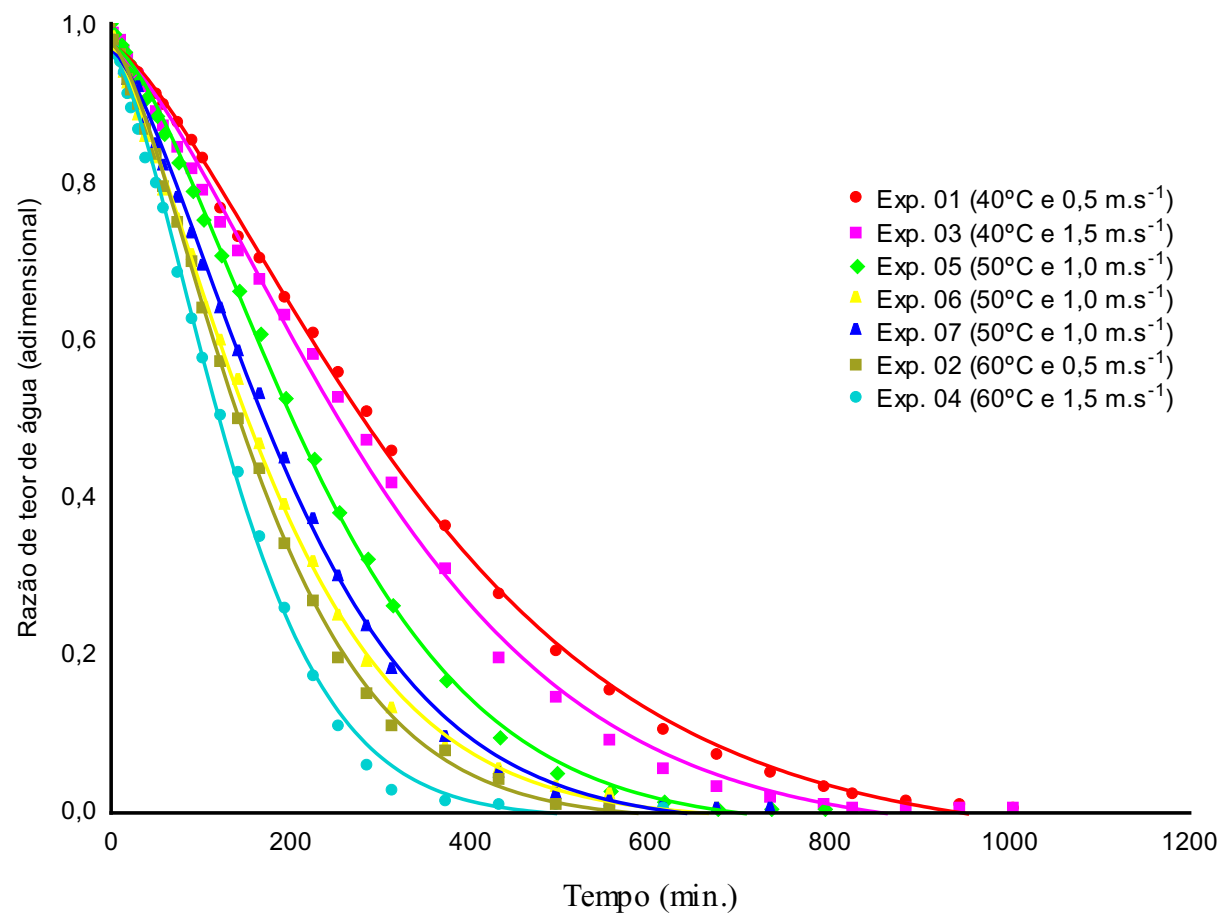

FIGURA 1. Equação de Midilli et al.(2002) ajustado aos dados experimentais da secagem de folhas de juazeiro

Conforme os resultados obtidos para os coeficientes de determinação, a equação de Midilli et al. (2002), utilizada foi a que melhor representou os dados experimentais para predizer a cinética de secagem das folhas de juazeiro, conforme se mostra na Figura 1, onde as curvas do modelo acompanham os pontos experimentais com pequenos desvios. A curva de Cavalcanti Mata (2006) apresentou excelentes resultados em relação aos coeficientes de determinação como também de DQM, porém a mesma não se ajustou aos dados experimentais.

Resultados semelhantes foram observados por Reis et al. (2011) que realizando a modelagem matemática de pimenta cumari nas temperaturas de 45,55 e $65^{\circ} \mathrm{C}$ concluíram que o modelo de Midilli et al. (2002) foi o que melhor representou a cinética de secagem da pimenta. Da mesma forma Martinazzo et al. (2007), ajustaram vários modelos matemáticos ao dados experimentais da secagem de folhas de capim - limão (Cymbopogon citratus) em camada delgada e concluíram que a equação de Midilli et al. (2002) entre os modelos avaliados, foi a que melhor se ajustou aos dados experimentais, apresentando o maior coeficiente de determinação.

Observou-se que em todos os tratamentos as equações matemáticas ajustadas aos dados experimentais, apresentaram coeficientes de determinação $\left(R^{2}\right)$ superiores a $97 \%$ e valores de DQM inferiores a 0,01(Tabela 3). Contudo, todas as equações podem ser usadas para o ajuste das curvas de secagem das folhas de juazeiro, nas condições de secagem estudadas. Em relação ao DQM, a equação de Cavalcanti Mata (2006) e Logarítmico (YAGCIOGLU et al., 1999) obtiveram os melhores resultados para todos os parâmetros estudados.

Em relação ao coeficiente de determinação $\left(R^{2}\right)$, as equações de aproximação por difusão (Corrêa et al, (2010), Cavalcanti Mata (2006), Logarítmico (YAGCIOGLU et al., 1999) Midilli et al. (2202), Page (Page, 1961) e Wang \& Sing (Wang \& Sing, 1978)presentaram valores elevados para todos os tratamentos, estando acima de $99 \%$. O bom ajuste das equações pode ser observado na Tabela 3. Comportamento similar foi verificado por Barbosa et al. (2007) que utilizando um secador de plantas medicinais, com as temperaturas de 40,50 , 60,70 e $80^{\circ} \mathrm{C}$ e velocidade de $0,29 \mathrm{~m} \mathrm{~s}^{-1}$ ajustaram os dados experimentais de erva-cidreira-brasileira (Lippia alba (Mill) N. E. Brown) várias equações e concluíram que as de Henderson \& Pabis (1961) Henderson \& Pabis modificado (1999) e Lewis (1921) foram as que melhor se ajustaram aos dados experimentais.

Martinazzo et al. (2010) estudando a modelagem matemática e os parâmetros qualitativos da secagem de folhas de capim-limão (Cymbopogon citratus)utilizaram equações matemáticas para descrever o processo de secagem e verificaram que a equação de Page Modificado (1999) o que melhor se ajusta aos dados experimentais. Portanto, a determinação do modelo matemático depende da espécie de planta estudada. 
TABELA 3. Resumo do ajuste dos dez modelos analisados por meio de regressão não linear aos dados experimentais da secagem de folhas de juazeiro em camada delgada

\begin{tabular}{|c|c|c|c|c|c|c|c|c|c|}
\hline Modelos & $\begin{array}{c}\mathrm{T} \\
\left({ }^{\circ} \mathrm{C}\right)\end{array}$ & $\begin{array}{c}\mathbf{V} \\
\left(\mathrm{m} \mathrm{s}^{-1}\right)\end{array}$ & $\begin{array}{c}\mathbf{R}^{2} \\
(\%)\end{array}$ & DQM & Modelos & $\begin{array}{c}\mathrm{T} \\
\left({ }^{\circ} \mathrm{C}\right)\end{array}$ & $\begin{array}{c}\mathbf{V} \\
\left(\mathrm{m} \mathrm{s}^{-1}\right)\end{array}$ & $\begin{array}{c}R^{2} \\
(\%)\end{array}$ & DQM \\
\hline & 40 & 0,5 & 99,65 & 0,0016 & & 40 & 0,5 & 99,48 & 0,0000 \\
\hline & 40 & 1,5 & 99,45 & 0,0018 & & 40 & 1,5 & 99,24 & 0,0000 \\
\hline Aproximação & 50 & 1,0 & 99,60 & 0,0016 & Logarítmico & 50 & 1,0 & 99,31 & 0,0000 \\
\hline \multirow[t]{6}{*}{ por difusão } & 50 & 1,0 & 99,76 & 0,0011 & & 50 & 1,0 & 99,35 & 0,0000 \\
\hline & 50 & 1,0 & 99,59 & 0,0017 & & 50 & 1,0 & 99,27 & 0,0000 \\
\hline & 60 & 0,5 & 99,54 & 0,0017 & & 60 & 0,5 & 98,94 & 0,0000 \\
\hline & 60 & 1,5 & 99,82 & 0,0010 & & 60 & 1,5 & 99,21 & 0,0000 \\
\hline & 40 & 0,5 & 99,39 & 0,0006 & & 40 & 0,5 & 99,91 & 0,0000 \\
\hline & 40 & 1,5 & 99,66 & 0,0004 & & 40 & 1,5 & 99,75 & 0,0001 \\
\hline Cavalcanti & 50 & 1,0 & 99,82 & 0,0081 & & 50 & 1,0 & 99,85 & 0,0000 \\
\hline \multirow[t]{6}{*}{ Mata } & 50 & 1,0 & 99,84 & 0,0000 & Midilli & 50 & 1,0 & 99,85 & 0,0000 \\
\hline & 50 & 1,0 & 99,89 & 0,0037 & & 50 & 1,0 & 99,92 & 0,0000 \\
\hline & 60 & 0,5 & 99,78 & 0,0003 & & 60 & 0,5 & 99,80 & 0,0001 \\
\hline & 60 & 1,5 & 99,80 & 0,0004 & & 60 & 1,5 & 99,92 & 0,0000 \\
\hline & 40 & 0,5 & 98,07 & 0,0018 & & 40 & 0,5 & 99,35 & 0,0043 \\
\hline & 40 & 1,5 & 97,95 & 0,0020 & & 40 & 1,5 & 99,45 & 0,0018 \\
\hline \multirow[t]{7}{*}{ Dois termos } & 50 & 1,0 & 97,64 & 0,0019 & & 50 & 1,0 & 99,65 & 0,0011 \\
\hline & 50 & 1,0 & 98,77 & 0,0016 & Page & 50 & 1,0 & 99,73 & 0,0013 \\
\hline & 50 & 1,0 & 98,18 & 0,0019 & & 50 & 1,0 & 99,60 & 0,0020 \\
\hline & 60 & 0,5 & 98,09 & 0,0022 & & 60 & 0,5 & 99,59 & 0,0019 \\
\hline & 60 & 1,5 & 98,41 & 0,0016 & & 60 & 1,5 & 99,83 & 0,0010 \\
\hline & 40 & 0,5 & 97,58 & 0,0001 & & 40 & 0,5 & 97,61 & 0,0003 \\
\hline & 40 & 1,5 & 97,45 & 0,0001 & & 40 & 1,5 & 97,49 & 0,0001 \\
\hline Exponencial & 50 & 1,0 & 98,88 & 0,0001 & & 50 & 1,0 & 96,84 & 0,0011 \\
\hline de dois & 50 & 1,0 & 98,38 & 0,0002 & Verma & 50 & 1,0 & 98,43 & 0,0002 \\
\hline \multirow[t]{4}{*}{ termos } & 50 & 1,0 & 98,69 & 0,0001 & & 50 & 1,0 & 97,73 & 0,0002 \\
\hline & 60 & 0,5 & 97,49 & 0,0001 & & 60 & 0,5 & 97,53 & 0,0001 \\
\hline & 60 & 1,5 & 97,78 & 0,0001 & & 60 & 1,5 & 97,76 & 0,0010 \\
\hline & 40 & 0,5 & 98,05 & 0,0018 & & 40 & 0,5 & 99,81 & 0,0006 \\
\hline Henderson & 40 & 1,5 & 97,95 & 0,0020 & & 40 & 1,5 & 99,75 & 0,0006 \\
\hline \& Pabis & 50 & 1,0 & 98,81 & 0,0019 & Wang \& Sing & 50 & 1,0 & 99,58 & 0,0014 \\
\hline \multirow[t]{4}{*}{ Modificado } & 50 & 1,0 & 99,38 & 0,0016 & & 50 & 1,0 & 99,73 & 0,0001 \\
\hline & 50 & 1,0 & 98,08 & 0,0019 & & 50 & 1,0 & 99,81 & 0,0005 \\
\hline & 60 & 0,5 & 99,04 & 0,0022 & & 60 & 0,5 & 99,68 & 0,0005 \\
\hline & 60 & 1,5 & 99,20 & 0,0016 & & 60 & 1,5 & 99,65 & 0,0058 \\
\hline
\end{tabular}

\section{CONCLUSÕES}

Quanto mais elevada à temperatura, mais rapidamente se processa a secagem das folhas de juazeiro.

A velocidade do ar de secagem apresentou influência sobre a taxa de secagem, apenas quando se trabalhou com a temperatura de $60^{\circ} \mathrm{C} \mathrm{e} 1,5 \mathrm{~m} \mathrm{~s}^{-1}$. Todos os modelos testados representaram satisfatoriamente os dados experimentais com coeficientes de determinação superior a $97 \%$, sendo o de Midilli et al. (2002) melhor.

\section{REFERÊNCIAS}

BARBOSA, F.F.; MELO, E.C.; SANTOS, R.H.S.; ROCHA, R. P.; MARTINAZZO, A. P.; RADÜNZ, L. L.; GRACIA, 
L. M. N. Evaluation of mathematical models for prediction of thinlayer drying of brazilian lemon-scented verbena leaves (Lippia alba (mill) N.E. Brown). Revista Brasileira de Produtos Agroindustriais, v.9, n.1, p.73-82, 2007.

BRASIL. Instituto Adolfo Lutz. Normas analíticas do Instituto Adolfo Lutz: Métodos químicos e físicos para análise de alimentos. $4^{a}$ ed. Instituto Adolfo Lutz, São Paulo, Brasil, 2005, 1018p.

CORREAA, P. C.; OLIVEIRA, G. H. H.; BOTELHO, F. M.; GONELI, A. L. D.; CARVALHO, F. M. Modelagem matemática e determinação das propriedades termodinâmicas do café (Coffea arabica L.) durante o processo de secagem. Revista Ceres, v.57, p.595601, 2010.

DIAS, R.A.L.; SOUZA, P.S.; ALSINA, O.L.S. Secagem e Extração de Taninos Totais da Hortelã (Mentha $x$ vilosa Hudson) Revista Agrarian, v.4, n.12, p.123133, 2011

FARIA, R.Q.; TEIXEIRA, I.R.; DEVILLA, I.A.; ASCHERI, D.P.R.; RESENDE, O. Cinética de secagem de sementes de crambe. Revista Brasileira de Engenharia Agrícola e Ambiental. v.16, n.5, p.573-583, 2012.

HENDERSON, S.M.; PABIS, S. Grain drying theory. Temperature effect on drying coefficient. Journal of Agricultural Engineering Research, n.6, p.169-174, 1961.

KARATHANOS, V.T. Determination of water content of dried fruits by drying kinetics. Journal of Food Engineering, v.39, p.337-344, 1999.

JITTANIT, W. Kinetics and temperature dependent moisture diffusivities of pumpkin seeds during drying. Kasetsart Journal: Natural Science, v.45, p.147-158, 2011.

LOPPES, A. H. Índice terapêutico fitoterápico. Petrópolis: EPUB, 2008.

MARTINAZZO, A.P.; MELO, E.C.; CORREAA, P.C.; SANTOS, R.H.S. Modelagem matemática e parâmetros qualitativos da secagem de folhas de capim-limão [Cymbopogon citratus (DC.) Stapf]. Revista Brasileira de Plantas Medicinais, v.12. n.4, p.488-498, 2010.

MARTINAZZO, A.P.; CORRÊA, P.C.; RESENDE, O.; MELO, E.C. Análise e descrição matemática da cinética de secagem de folhas de capim-limão. Revista Brasileira de Engenharia Agropecuária, v.11, n.3, p.301-306, 2007.

MIDILLI, A.; KUCUK, H.; YAPAR, Z.A. A new model for single-layer drying. Drying Technology, v.20, n.7, p.1503-1513, 2002.

PAGE, G. E. Factors influencing the maximum rates of air drying shelled corn in thin layers. 1949. Thesis (M.Sc.) - Purdue University, West Lafayette, 1949.

RADUNZ, L.L.; AMARAL, A.S.; MOSSI, S.J.; MELO, E.C.; ROCHA, R.P. Avaliação da cinética de secagem da carqueja. Revista Engenharia na Agricultura, v.19, n.1, p.19-27, 2011.

RADUNZ, L.L.; MOSSI, A.J.; ZAKRZEVSKI.; C.A.; AMARAL, A.S,R,P.; GRASSMANN, L. Análise da cinética de secagem de folhas de sálvia. Revista Brasileira de Engenharia Agrícola e Ambiental, v.14, n.9, p.979-986, 2010.

REIS, R.C.; BARBOSA, L.S.; LIMA, M.L.; REIS, J.S.; DEVILLA, I.A.; ASCHERI, D.P.R. Modelagem matemática da secagem da pimenta Cumari do Pará. Revista Brasileira de Engenharia Agrícola e Ambiental. v.15, n.4, p.347-533, 2011.

RESENDE, O.; CORREAA, P.C.; GONELI, A.L.D.; BOTELHO, F.M.; RODRIGUES, S. Modelagem matemática do processo de secagem de duas variedades de feijão (Phaseolus vulgaris L.). Revista Brasileira de Produtos Agroindustriais, v.10, n.1, p.17-26, 2008.

SHARAF-ELDEEN, Y. I.; BLAISDELL, J. L.; HAMDY, M. Y. A model for ear corn drying. Transactions of the ASAE, v.23, p.1.261-1.265, 1980.

WANG, C.Y.; SINGH, R.P. Use of variable equilibrium moisture content in modeling rice drying. Transaction of ASAE, v.11, n.A, 1978.

YAGCIOGLU, A.; DEGIRMENCIOGLU, A.; CAGATAY, F. Drying characteristics of laurel leaves under different conditions. In: INTERNATIONAL CONGRESS ON AGRICULTURAL MECHANIZATION AND ENERGY, 7, 1999.

VERMA, L.R.; BUCKLIN, R.A.; ENDAN, J.B.; WRATTEN, F.T. Effects of drying air parameters on rice drying models. Transactions of the ASAE, v.28, p.296-301, 1985. 